\title{
Madridge Journal of
}

madridge Bioinformatics and Systems Biology

Interconnecting Scientific World

Research Article

Open Access

\section{Common fixed point theorem in intuitionistic Fuzzy metric Spaces using compatible mappings of type (A)}

\section{Saurabh Manro*}

School of Mathematics and Computer Applications, Thapar University, Patiala, India

\section{Article Info}

\author{
*Corresponding author: \\ Saurabh Manro \\ School of Mathematics and Computer \\ Applications \\ Thapar University \\ Patiala, India \\ E-mail: sauravmanro@hotmail.com
}

Received: December 5, 2018

Accepted: December 11, 2018

Published: December 17, 2018

Citation: Manro S. Common fixed point theorem in intuitionistic Fuzzy metric Spaces using compatible mappings of type (A). Madridge J Bioinform Syst Biol. 2018; 1(1): 5-9.

doi: $10.18689 /$ mjbsb-1000102

Copyright: (c) 2018 The Author(s). This work is licensed under a Creative Commons Attribution 4.0 International License, which permits unrestricted use, distribution, and reproduction in any medium, provided the original work is properly cited.

Published by Madridge Publishers

\begin{abstract}
In this paper, we prove common fixed point theorem in intuitionistic fuzzy metric space using compatible mappings of type $(A)$.
\end{abstract}

Keywords: Intuitionistic Fuzzy metric space; Compatible mappings of type (A); Common fixed point.

AMS (2010) Subject Classification: 47H10, 54H25

\section{Introduction}

Atanassove [2] introduced and studied the concept of intuitionistic fuzzy sets as a generalization of fuzzy sets. In 2004, Park [5] defined the notion of intuitionistic fuzzy metric space with the help of continuous t-norms and continuous t-conorms. Recently, in 2006, Alaca et al.[1] using the idea of Intuitionistic fuzzy sets, defined the notion of intuitionistic fuzzy metric space with the help of continuous t-norm and continuous tconorms as a generalization of fuzzy metric space due to Kramosil and Michalek [3]. In this paper, we prove common fixed point theorem in intuitionistic fuzzy metric space using compatible mappings of type (A).

\section{Preliminaries}

The concepts of triangular norms (t-norms) and triangular conorms (t-conorms) are known as the axiomatic skelton that we use are characterization fuzzy intersections and union respectively. These concepts were originally introduced by Menger [4] in study of statistical metric spaces.

Definition [6] A binary operation *: $[0,1] \times[0,1] \rightarrow[0,1]$ is continuous t-norm if * satisfies the following conditions:
(i) * is commutative and associative;
(ii) * is continuous;
(iii) $a * 1=$ a for all $a \in[0,1]$;
(iv) $\mathrm{a} * \mathrm{~b} \leq \mathrm{c} * \mathrm{~d}$ whenever $\mathrm{a} \leq \mathrm{c}$ and $\mathrm{b} \leq \mathrm{d}$ for all $a, b, c, d \in[0,1]$.

Definition [6] A binary operation $\diamond:[0,1] \times[0,1] \rightarrow[0,1]$ is continuous t-conorm if $\diamond$ satisfies the following conditions:

(i) $\diamond$ is commutative and associative;

(ii) $\diamond$ is continuous;

(iii) $a \diamond 0=$ a for all $a \in[0,1]$; 
(iv) $\mathrm{a} \diamond \mathrm{b} \leq \mathrm{c} \diamond \mathrm{d}$ whenever $\mathrm{a} \leq \mathrm{c}$ and $\mathrm{b} \leq \mathrm{d}$ for all $a, b, c$, $d \in[0,1]$.

Alaca et al. [1] using the idea of Intuitionistic fuzzy sets, defined the notion of intuitionistic fuzzy metric space with the help of continuous t-norm and continuous t-conorms as a generalization of fuzzy metric space due to Kramosil and Michalek [3] as:

Definition [1] A 5-tuple $(X, M, N, *, \diamond)$ is said to be an intuitionistic fuzzy metric space if $X$ is an arbitrary set, * is a continuous t-norm, $\diamond$ is a continuous t-conorm and $M, N$ are fuzzy sets on $X^{2} \times[0, \infty)$ satisfying the following conditions:

(i) $\mathrm{M}(\mathrm{x}, \mathrm{y}, \mathrm{t})+\mathrm{N}(\mathrm{x}, \mathrm{y}, \mathrm{t}) \leq 1$ for all $x, y \in X$ and t $>0$;

(ii) $\mathrm{M}(\mathrm{x}, \mathrm{y}, 0)=0$ for all $x, y \in X$;

(iii) $\mathrm{M}(\mathrm{x}, \mathrm{y}, \mathrm{t})=1$ for all $x, y \in X$; and $\mathrm{t}>0$ if and only if $\mathrm{x}=\mathrm{y}$;

(iv) $\mathrm{M}(\mathrm{x}, \mathrm{y}, \mathrm{t})=\mathrm{M}(\mathrm{y}, \mathrm{x}, \mathrm{t})$ for all $x, y \in X$ and $\mathrm{t}>0$;

(v) $M(x, y, t) * M(y, z, s) \leq M(x, z, t+s)$ for all $x, y, z \in X$ and $s, t>0$;

(vi) for all $x, y \in X, M(x, y,):.[0, \infty) \rightarrow[0,1]$ is left continuous;

(vii) $\lim _{\mathrm{t} \rightarrow \infty} \mathrm{M}(\mathrm{x}, \mathrm{y}, \mathrm{t})=1$ for all $x, y \in X$ and $\mathrm{t}>0$;

(viii) $\mathrm{N}(x, y, 0)=1$ for all $x, y \in X$;

(ix) $\mathrm{N}(\mathrm{x}, \mathrm{y}, \mathrm{t})=0$ for all $x, y \in X$ and $\mathrm{t}>0$ if and only if $\mathrm{x}=\mathrm{y}$;

(x) $\quad \mathrm{N}(\mathrm{x}, \mathrm{y}, \mathrm{t})=\mathrm{N}(\mathrm{y}, \mathrm{x}, \mathrm{t})$ for all $x, y \in X$ and $\mathrm{t}>0$;

(xi) $\mathrm{N}(\mathrm{x}, \mathrm{y}, \mathrm{t}) \diamond \mathrm{N}(\mathrm{y}, \mathrm{z}, \mathrm{s}) \geq \mathrm{N}(\mathrm{x}, \mathrm{z}, \mathrm{t}+\mathrm{s})$ for all $x, y, z \in X$ and $s, t>0$;

(xii) for all $x, y \in X, N(x, y,):.[0, \infty) \rightarrow[0,1]$ is right continuous; (xiii) $\lim _{\mathrm{t} \rightarrow \infty} \mathrm{N}(\mathrm{x}, \mathrm{y}, \mathrm{t})=0$ for all $x, y \in X$.

Then $(M, N)$ is called an intuitionistic fuzzy metric space on $X$. The functions $M(x, y, t)$ and $N(x, y, t)$ denote the degree of nearness and the degree of non-nearness between $x$ and $y$ w.r.t. $t$ respectively.

Remark 2.1: Every fuzzy metric space $\left(X_{1}, M^{*}{ }^{*}\right)$ is an intuitionistic fuzzy metric space of the form $(X, M, 1-M, *, \diamond)$ such that t-norm * and t-conorm $\diamond$ are associated as $\mathrm{x} \diamond \mathrm{y}=$ $1-((1-\mathrm{x}) *(1-\mathrm{y}))$ for all $x, y \in X$.

Remark 2.2: In intuitionistic fuzzy metric space $(X, M, N$, *, $\diamond), M(x, y, *)$ is non-decreasing and $N(x, y, \diamond)$ is non-increasing for all $x, y \in X$.

Alaca, Turkoglu and Yildiz [1] introduced the following notions:

Definition Let $\left(X, M, N,{ }^{*}, \diamond\right)$ be an intuitionistic fuzzy metric space. Then

(a) a sequence $\left\{x_{n}\right\}$ in $X$ is said to be Cauchy sequence if, for all $t>0$ and $p>0, \lim _{n \rightarrow \infty} M\left(x_{n+p^{\prime}}, x_{n^{\prime}} t\right)=1$ and $\lim _{n \rightarrow \infty} N\left(x_{n+p^{\prime}}\right.$ $\left.\mathrm{x}_{\mathrm{n}^{\prime}} \mathrm{t}\right)=0$.

(b) a sequence $\left\{x_{n}\right\}$ in $X$ is said to be convergent to a point $x \in X$ if, for all $t>0, \lim _{n \rightarrow \infty} M\left(x_{n^{\prime}} x, t\right)=1$ and $\lim _{n \rightarrow \infty} N\left(x_{n^{\prime}}, x\right.$, $\mathrm{t})=0$.

Definition [1] an intuitionistic fuzzy metric space $(X, M, N, *$, $\diamond)$ is said to be complete if and only if every Cauchy sequence in $\mathrm{X}$ is convergent.
Example 2.1: Let $X=\{1 / n: n \in N\} \cup\{0\}$ and let * be the continuous t-norm and $\diamond$ be the continuous t-conorm defined by $\mathrm{a} * \mathrm{~b}=\mathrm{ab}$ and $\mathrm{a} \diamond \mathrm{b}=\min \{1, \mathrm{a}+\mathrm{b}\}$ respectively, for all $a, b$ $\in[0,1]$. For each $t \in(0, \infty)$ and $x, y \in X$, define $(M, N)$ by

$M(x, y, t)=\left\{\begin{array}{ccc}\frac{t}{t+|x-y|}, & t>0, \\ 0 & t=0\end{array} \quad\right.$ and $N(x, y, t)=\left\{\begin{array}{cc}\frac{|x-y|}{t+|x-y|} & t>0 \\ 1 & t=0\end{array}\right.$

Clearly, $(X, M, N, *, \diamond)$ is complete intuitionistic fuzzy metric space.

Definition A pair of self mappings $(f, g)$ of a intuitionistic fuzzy metric space $(X, M, N, *$,$) is said to be compatible if$ $\lim _{n \rightarrow \infty} M\left(f g x_{n^{\prime}} g f x_{n^{\prime}} t\right)=1$ and $\lim _{n \rightarrow \infty} N\left(f g x_{n^{\prime}} g f x_{n^{\prime}} t\right)=0$ for all $t$ $>0$, whenever $\left\{x_{n}\right\}$ is a sequence in $X$ such that $\lim _{n \rightarrow \infty} f x_{n}=$ $\lim _{n \rightarrow \infty} g x_{n}=u$ for some $u$ in $X$.

Definition A pair of self mappings ( $f, g$ ) of a intuitionistic fuzzy metric space $\left(X, M, N,{ }^{*}, \nabla\right)$ is said to be compatible of type $(A)$ iflim $_{n \rightarrow \infty} M\left(f g x_{n^{\prime}} g g x_{n^{\prime}} t\right)=1, \lim _{n \rightarrow \infty} N\left(f g x_{n^{\prime}} g g x_{n^{\prime}} t\right)=0$ and $\lim _{n \rightarrow \infty} M\left(g f x_{n^{\prime}} f f x_{n^{\prime}} t\right)=1, \lim _{n \rightarrow \infty} N\left(g f x_{n^{\prime}} f f x_{n^{\prime}} t\right)=0$.

for all $t>0$, whenever $\left\{x_{n}\right\}$ is a sequence in $X$ such that $\lim _{n \rightarrow \infty} f x_{n}$ $=\lim _{n \rightarrow \infty} g x_{n}=u$ for some $u$ in $X$.

\section{Alaca [1] proved the following results:}

Lemma Let $\left(X, M, N,{ }^{*}, \diamond\right)$ be intuitionistic fuzzy metric space and for all $\mathrm{x}, \mathrm{y}$ in $\mathrm{X}, \mathrm{t}>0$ and if for a number $k>1$ such that

$M(x, y, k t) \leq M(x, y, t)$ and $N(x, y, k t) \geq N(x, y, t)$ Then $x=y$.

Lemma Let $\left(X, M, N,{ }^{*}, \diamond\right)$ be intuitionistic fuzzy metric space and for all $\mathrm{x}, \mathrm{y}$ in $\mathrm{X}, \mathrm{t}>0$ and if for a number $k>1$ such that

$M\left(y_{n+2^{\prime}}, y_{n+1^{\prime}} t\right) \geq M\left(y_{n+1^{\prime}} y_{n^{\prime}} k t\right), N\left(y_{n+2^{\prime}} y_{n+1^{\prime}} t\right) \leq N\left(y_{n+1}, y_{n^{\prime}} k t\right)$.

Then $\left\{y_{n}\right\}$ is a Cauchy sequence in $X$.

Lemma Let $\mathrm{f}$ and $\mathrm{g}$ be compatible self mappings of type (A) of a complete intuitionistic fuzzy metric space $\left(X, M, N,{ }^{*}, \diamond\right)$ with $a^{*} b=\min \{a, b\}$ and $a \diamond b=\max \{a, b\}$ for all $a, b \in[0,1]$ and $\mathrm{fu}=\mathrm{gu}$ for some $u \in X$. Then $\mathrm{gfu}=\mathrm{fgu}=\mathrm{ffu}=\mathrm{ggu}$.

\section{Results}

Theorem: Let $\left(X, M, N^{*}, \oslash\right)$ be a complete intuitionistic fuzzy metric space with $a^{*} b=\min \{a, b\}$ and $a \diamond b=\max \{a, b\}$ for all $a, b \in[0,1]$. Let $\mathrm{A}, \mathrm{B}, \mathrm{S}, \mathrm{T}, \mathrm{P}$ and $\mathrm{Q}$ be mappings from $\mathrm{X}$ into itself such that the following conditions are satisfied:

(3.1) $P(X) \subseteq S T(X), Q(X) \subseteq A B(X)$,

(3.2) $\mathrm{AB}=\mathrm{BA}, \mathrm{ST}=\mathrm{TS}, \mathrm{PB}=\mathrm{BP}, \mathrm{QT}=\mathrm{TQ}$,

(3.3) $P$ or $A B$ is continuous,

(3.4) $(P, A B)$ and $(Q, S T)$ are pairs of compatible mappings of type $(A)$,

(3.5) there exist $k \in(0,1)$ such that for every $x, y \in X$ and $t>0$ $M(P x, Q y, k t) \geq M(A B x, S T y, t) * M(P x, A B x, t)$ * $M(Q y, S T y, t)$ * $M(P x, S T y, t)$

$N(P x, Q y, k t) \leq N(A B x, S T y, t) \diamond N(P x, A B x, t) \diamond N(Q y, S T y, t) \diamond N$ 
$(P x, S T y, t)$

Then $A, B, S, T, P$ and $Q$ have a unique common fixed point in $\mathrm{x}$.

\section{Proof}

\section{Forexistence:}

Let $x_{0} \in X_{2^{\prime}}$ from (3.1), there exist $x_{1}, x_{2} \in X$ such that

$P x_{0}=S T x_{1}, Q x_{1}=A B x_{2}$. Inductively, we Construct sequences $\left\{x_{n}\right\}$ and $\left\{y_{n}\right\}$ in $X$ such that

$P X_{2 n-2}=S T X_{2 n-1}=y_{2 n-1}$ and $Q x_{2 n-1}=A B x_{2 n}=y_{2 n}$

for $n=1,2,3, \ldots$

Take $x=x_{2 n}, y=x_{2 n}+$ in (3.5), we get

$M\left(P x_{2 n^{\prime}} Q x_{2 n+11^{\prime}} k t\right) \geq M\left(A B x_{2 n^{\prime}} S T x_{2 n+1} t\right)$ *M(Px $\left.2 x_{2 n^{\prime}} A B x_{2 n^{\prime}} t\right)$ *M $\left(Q x_{2 n+1}, S T x_{2 n+1}, t\right) * M\left(P x_{2 n^{\prime}} S T x_{2 n+1} t\right)$

$M\left(y_{2 n+1^{\prime}} y_{2 n+2^{\prime}} k t\right) \geq M\left(y_{2 n^{\prime}} y_{2 n+1^{\prime}} t\right) * M\left(y_{2 n+1^{\prime}}, y_{2 n,} t\right) * M\left(y_{2 n+2,}\right.$ $\left.y_{2 n+1^{\prime}} t\right)^{*} M\left(y_{2 n+1}, y_{2 n+22^{\prime}} t\right)$

$M\left(y_{2 n+1^{\prime}} y_{2 n+2^{\prime}} k t\right) \geq M\left(y_{2 n^{\prime}} y_{2 n+1^{\prime}} t\right) * M\left(y_{2 n+1}, y_{2 n+2^{\prime}} t\right)$

$M\left(y_{2 n+1}, y_{2 n+2^{\prime}} k t\right) \geq M\left(y_{2 n^{\prime}} y_{2 n+1}, t\right)$

And

$N\left(P x_{2 n^{\prime}} Q x_{2 n+1}, k t\right) \leq N\left(A B x_{2 n^{\prime}} S T x_{2 n+1} t\right) \diamond N\left(P x_{2 n^{\prime}} A B x_{2 n^{\prime}} t\right) \diamond N$ $\left(Q x_{2 n+1}, S T x_{2 n+1,} t\right) \otimes N\left(P x_{2 n^{\prime}}, S T x_{2 n+1}, t\right)$

$N\left(y_{2 n+1}, y_{2 n+2^{\prime}} k t\right) \leq N\left(y_{2 n^{\prime}} y_{2 n+1}, t\right) \diamond N\left(y_{2 n+1}, y_{2 n^{\prime}} t\right) \diamond N\left(y_{2 n+22^{\prime}} y_{2 n+11^{\prime}}\right.$ $t) \diamond N\left(y_{2 n+1}, y_{2 n+2,}, t\right)$

$N\left(y_{2 n+1}, y_{2 n+2^{\prime}}, t\right) \leq N\left(y_{2 n^{\prime}} y_{2 n+1}, t\right) \diamond N\left(y_{2 n+1^{\prime}} y_{2 n+2^{\prime}} t\right)$

$N\left(y_{2 n+1}, y_{2 n+2}, k t\right) \leq N\left(y_{2 n^{\prime}}, y_{2 n+11^{\prime}}, t\right)$

Similarly, $M\left(y_{2 n+2^{\prime}} y_{2 n+3^{\prime}} k t\right) \geq M\left(y_{2 n+1^{\prime}} y_{2 n+2^{\prime}} t\right), N\left(y_{2 n+2^{\prime}} y_{2 n+3^{\prime}} k t\right)$ $\leq N\left(y_{2 n+1}, y_{2 n+2^{\prime}} t\right)$.

Thus, we have

$M\left(y_{n+1^{\prime}} y_{n+2^{\prime}}, k t\right) \geq M\left(y_{n^{\prime}} y_{n+1^{\prime}}, t\right), N\left(y_{n+1^{\prime}} y_{n+2^{\prime}} k t\right) \leq N\left(y_{n^{\prime}} y_{n+1^{\prime}} t\right)$ for $\mathrm{n}=1,2,3, \ldots$.

Therefore, we have

$M\left(y_{n^{\prime}} y_{n+1} t\right) \geq M\left(y_{n^{\prime}} y_{n+1^{\prime}} t / q\right) \geq M\left(y_{n-1^{\prime}} y_{n^{\prime}} t / q^{2}\right) \geq \ldots \geq M\left(y_{1^{\prime}} y_{2^{\prime}}\right.$ $\left.t / q^{n}\right) \rightarrow 1$,

$N\left(y_{n^{\prime}} y_{n+1}, t\right) \leq N\left(y_{n^{\prime}} y_{n+1}{ }^{\prime} t / q\right) \leq N\left(y_{n-1}, y_{n^{\prime}} t / q^{2}\right) \leq \ldots \leq N\left(y_{1^{\prime}}, y_{2^{\prime}} t\right)$ $\left.q^{n}\right) \rightarrow 0$

when $n \rightarrow \infty$.

For each $\in>0$ and $t>0$, we can choose $n_{0} \in \mathrm{N}$ such that

$M\left(y_{n^{\prime}} y_{n+1}, t\right)>1-\in, N\left(y_{n^{\prime}}, y_{n+1}, t\right)<\in$ for all $n \geq n_{0^{\prime}}$.

For $m, n \in \mathbb{N}$, we suppose $m \geq n$. Then, we have

$M\left(y_{n^{\prime}} y_{m^{\prime}} t\right) \geq M\left(y_{n^{\prime}} y_{n+1} t / m-n\right)^{\star} M\left(y_{n+1} y_{n+2^{\prime}} t / m-n\right)^{*} \ldots{ }^{*} M$

$\left(y_{\mathrm{m}-1} y_{\mathrm{m}^{\prime}} t / m-n\right)$

$>\left((1-\epsilon){ }^{*}(1-\epsilon) * . . *(1-\epsilon)\right)(m-n)$ times

$\geq(1-\epsilon)$
$N\left(y_{n^{\prime}} y_{m^{\prime}} t\right) \leq N\left(y_{n^{\prime}} y_{n+1^{\prime}} t / m-n\right) \diamond N\left(y_{n+1^{\prime}} y_{n+2^{\prime}} t / m-n\right) \diamond \ldots \diamond N$ $\left(y_{m-1} y_{m^{\prime}} t / m-n\right)$

$<((\in) \diamond(\in) \diamond \ldots \diamond(\in))(m-n)$ times

$\leq(\epsilon) M\left(y_{\mathrm{n}^{\prime}} y_{\mathrm{m}^{\prime}} t\right)>(1-\epsilon), N\left(y_{\mathrm{n}^{\prime}} y_{\mathrm{m}^{\prime}} t\right)<\epsilon$

and hence $\left\{y_{n}\right\}$ is a Cauchy sequence in $X$. As $X$ is complete, $\left\{y_{n}\right\}$ converges to some point

$z \in X$. Also, its subsequences converge to this point $z \in X$.

i.e. $\left\{Q x 2_{n+1}\right\} \rightarrow z_{,}\left\{S T x_{2 n+1}\right\} \rightarrow z_{1}\left\{P x_{2 n}\right\} \rightarrow z_{1}\left\{A B x_{2 n}\right\} \rightarrow z$.

Suppose $A B$ is continuous.

As $A B$ is continuous, we have $\left\{A B A B x_{2 n}\right\} \rightarrow A B z,\left\{A B P x_{2 n}\right\} \rightarrow A B z$.

As $(P, A B)$ is compatible pair of type $(A)$, we have $\left\{P A B x_{2 n}\right\} \rightarrow$ $A B z$.

Take $x_{=} A B x_{2 n^{\prime}} y_{=} x_{2 n+1}$ in (3.5), we get

$M\left(P A B x_{2 n^{\prime}} Q x_{2 n+11^{\prime}} k t\right) \geq M\left(A B A B x_{2 n^{\prime}} S T x_{2 n+1^{\prime}} t\right) * M\left(P A B x_{2 n^{\prime}}\right.$ $\left.A B A B x_{2 n^{\prime}} t\right) * M\left(Q x_{2 n+1} S T x_{2 n+1}, t\right) * M\left(P A B x_{2 n^{\prime}} S T x_{2 n+11^{\prime}} t\right)$

$n_{\rightarrow \infty}$

$M(A B z, z, k t) \geq M(A B z, z, t) * M(A B z, A B z, t) * M(z, z, t)$ *

$M(A B z, z, t) M(A B z, z, k t) \geq M(A B z, z, t)$

And

$N\left(P A B x_{2 n^{\prime}} Q x_{2 n+1}, k t\right) \leq N\left(A B A B x_{2 n^{\prime}} S T x_{2 n+1,} t\right){ }_{0} N\left(P A B x_{2 n^{\prime}}\right.$ $\left.A B A B x_{2 n^{\prime}} t\right){ }_{\diamond} N\left(Q x_{2 n+1}, S T x_{2 n+1}, t\right){ }_{\diamond} N\left(P A B x_{2 n^{\prime}} S T x_{2 n+1} t\right)$

$n_{\rightarrow \infty}$

$N(A B z, z, k t){ }_{\leq} N(A B z, z, t){ }_{\diamond} N(A B z, A B z, t){ }_{\diamond} N(z, z, t) \diamond$

$N(A B z, z, t) N(A B z, z, k t) \leq N(A B z, z, t)$

By lemma, $A B z=z$.

Next, we show that $\mathrm{Pz}=\mathrm{z}$.

Put $x=z$ and $y=x_{2 n}$ in (3.5), we get

$M\left(P z_{1} Q x_{2 n^{\prime}} k t\right) \geq M\left(A B z_{1} S T x_{2 n^{\prime}} t\right) * M\left(P z_{1} A B z_{1} t\right) * M\left(Q x_{2 n^{\prime}} S T x_{2 n,}\right.$ $t) * M\left(P z_{1} S T x_{2 n^{\prime}} t\right)$

$n_{\rightarrow \infty}$

$M(P z, z, k t){ }_{\geq} M(z, z, t) * M(P z, z, t) * M(z, z, t) * M(P z, z, t) M$ $(P z, z, k t) \geq M(P z, z, t)$

And

$N\left(P z_{1} Q x_{2 n^{\prime}} k t\right){ }_{\leq} N\left(A B z, S T x_{2 n^{\prime}} t\right){ }_{\diamond} N(P z, A B z, t){ }_{\diamond} N\left(Q x_{2 n^{\prime}} S T x_{2 n^{\prime}}\right.$ t) ${ }_{\diamond} N\left(P Z, S T x_{2 n^{\prime}} t\right)$

$n_{\rightarrow \infty}$

$N(P z, z, k t){ }_{\leq} N(z, z, t){ }_{\diamond} N(P z, z, t){ }_{\diamond} N(z, z, t){ }_{\diamond} N(P z, z, t)$

$N(P z, z, k t){ }_{\leq} N(P z, z, t)$

Therefore, $A B z=z=P z$.

Now, we show that $B z=z$.

Put $\mathrm{x}=\mathrm{Bz}$ and $y_{=} x_{2 \mathrm{n}-1}$ in (3.5), we get

$M\left(P B z_{1} Q x_{2 n-1}, k t\right) \geq M\left(A B B z, S T x_{2 n-1}, t\right) * M(P B z, A B B z, t) * M$ 
$\left(Q x_{2 n-1^{\prime}} S T x_{2 n-1^{\prime}} t\right) * M\left(P B z_{1} S T x_{2 n-1,} t\right)$

$N\left(P B z, Q x_{2 \mathrm{n}-1^{\prime}} k t\right) \leq N\left(A B B z, S T x_{2 \mathrm{n}-1^{\prime}} t\right){ }_{\diamond} N(P B z, A B B z, t) \diamond N$ $\left(Q x_{2 n-1} S T x_{2 n-1,} t\right) \diamond N\left(P B z_{1} S T x_{2 n-1,} t\right)$

As $B P=P B$ and $A B=B A$, so that

$P(B z)=(P B) z=B P z=B z$ and $(A B)(B z)=(B A)(B z)=B(A B) z=B z$.

Taking, $n \rightarrow \infty$, we get

$M(B z, z, k t) \geq M(B z, z, t)$ * $M(B z, B z, t)$ * $M(z, z, t)$ * $M(B z, z, t)$

$M(B z, z, k t) \geq M(B z, z, t)$

And

$N(B z, z, k t) \leq N(B z, z, t) \diamond N(B z, B z, t) \diamond N(z, z, t) \diamond N(B z, z, t)$

$N(B z, z, k t) \leq N(B z, z, t)$.

Therefore, by using lemma, we get $B z=z$ and also we have, $A B z=z$. Therefore, $A z=B z=P z=z$.

As $P(X) \subseteq S T(X)$, there exist $u \in X$ such that $z=P z=S T u$.

Putting, $x=x_{2 \mathrm{n}}, y=u$ in (3.5), we get

$M\left(P x_{2 n^{\prime}} Q u, k t\right) \geq M\left(A B x_{2 n^{\prime}} S T u, t\right)$ *M(Px $\left.{ }_{2 n^{\prime}} A B x_{2 n^{\prime}} t\right)$ *M(Qu, $S T u, t) * M\left(P x_{2 n^{\prime}} S T u, t\right)$

$n \rightarrow \infty$

$M(z, Q u, k t) \geq M(z, z, t) * M(z, z, t) * M(Q u, z, t) * M(z, z, t)$

$M(z, Q u, k t) \geq M(Q u, z, t)$

And

$N\left(P x_{2 n^{\prime}} Q u, k t\right) \leq N\left(A B x_{2 n^{\prime}} S T u, t\right) \diamond N\left(P x_{2 n^{\prime}} A B x_{2 n^{\prime}} t\right) \diamond N(Q u$, $S T u, t) \diamond N\left(P x_{2 n^{\prime}} S T u, t\right)$

$n \rightarrow \infty N$

$(z, Q u, k t) \leq N(z, z, t) \diamond N(z, z, t) \diamond N(Q u, z, t) \diamond N(z, z, t)$

$N(z, Q u, k t) \leq N(z, Q u, t)$

By using lemma, we get $\mathrm{Qu}=\mathrm{z}$. Hence, $\mathrm{STu}=\mathrm{z}=\mathrm{Qu}$.

Since $(Q, S T)$ is compatible pair of type $(A)$, therefore, by lemma, we have QSTu = STQu. Therefore, Qz = STz.

Now, we show that $\mathrm{Qz}=\mathrm{z}$.

Take $x=x_{2 n,} y=z$ in (3.5), we get

$M\left(P x_{2 n^{\prime}} Q z, k t\right) \geq M\left(A B x_{2 n^{\prime}} S T z, t\right)$ * $M\left(P x_{2 n^{\prime}} A B x_{2 n^{\prime}} t\right)$ * $M(Q z$, $S T z, t) * M\left(P x_{2 n^{\prime}} S T z, t\right)$

$n \rightarrow \infty$

$M(z, Q z, k t) \geq M(z, Q z, t)$ * $M(z, z, t)$ * $M(Q z, Q z, t)$ * $M(z, Q z$, t)

$M(z, Q z, k t) \geq M(z, Q z, t)$

and

$N\left(P x_{2 n^{\prime}} Q z, k t\right) \leq N\left(A B x_{2 n^{\prime}} S T z, t\right) \diamond N\left(P x_{2 n^{\prime}} A B x_{2 n^{\prime}} t\right) \diamond N(Q z, S T z$, t) $\diamond N\left(P x_{2 n^{\prime}} S T z, t\right)$

$n \rightarrow \infty$

$N(z, Q z, k t) \leq N(z, Q z, t) \diamond N(z, z, t) \diamond N(Q z, Q z, t) \diamond N(z, Q z, t)$
$N(z, Q z, k t) \leq N(z, Q z, t)$

Therefore, by using lemma, $Q z=z$.

As QT = TQ, ST = TS, we have QTz = TQz = Tz and STTz = TSTz $=T Q z=T z$.

Next, we claim that $T z=z$.

For this, take $=x_{2 n}, y=T z$ in (3.5), we get

$M\left(P x_{2 n^{\prime}} Q T z, k t\right) \geq M\left(A B x_{2 n^{\prime}} S T T z, t\right)$ *M(Px $\left.2 n^{\prime} A B x_{2 n^{\prime}} t\right)$ *M(Qz, $S T T z, t) * M\left(P x_{2 n^{\prime}} S T T z, t\right)$

$n \rightarrow \infty$

$M(z, T z, k t) \geq M(z, T z, t) * M(z, z, t) * M(T z, T z, t) * M(z, T z, t)$

$M(z, T z, k t) \geq M(z, T z, t)$

and

$N\left(P x_{2 n^{\prime}} Q T z, k t\right) \leq N\left(A B x_{2 n^{\prime}} S T T z_{1} t\right) \diamond N\left(P x_{2 n^{\prime}} A B x_{2 n^{\prime}} t\right) \diamond N(Q T z$, $S T T z, t) \diamond N\left(P x_{2 n^{\prime}} S T T z, t\right)$

$n \rightarrow \infty$

$N(z, T z, k t) \leq N(z, T z, t) \diamond N(z, z, t) \diamond N(T z, T z, t) \diamond N(z, T z, t)$

$N(z, T z, k t) \leq N(z, T z, t)$

therefore, by lemma, we get $\mathrm{Tz}=\mathrm{z}$. as $\mathrm{STz}=\mathrm{Qz}=\mathrm{z}=\mathrm{Tz}$. This gives, $\mathrm{Sz}=\mathrm{z}$. Hence, $\mathrm{Az}=\mathrm{Bz}=\mathrm{Pz}=\mathrm{Qz}=\mathrm{Sz}=\mathrm{T} z=\mathrm{z}$. Hence, $z$ is a common fixed point of $A, B, S, T, P$ and $Q$. The proof is similar $\mathrm{P}$ is continuous.

\section{For uniqueness}

Let $\mathrm{u}$ is another fixed point of $\mathrm{A}, \mathrm{B}, \mathrm{S}, \mathrm{T}, \mathrm{P}$ and $\mathrm{Q}$. Therefore, take $x=z$ and $y=u$ in (3.5), we get

$M(P z, Q u, k t) \geq M(A B z, S T u, t)$ * $M(P z, A B z, t)$ * $M(Q u, S T u, t)$ * $M(P z, S T u, t)$

$M(z, u, k t) \geq M(z, u, t) * M(z, z, t) * M(u, u, t) * M(z, u, t)$

$M(z, u, k t) \geq M(z, u, t)$

And $N(P z, Q u, k t) \leq N(A B z, S T u, t) \diamond N(P z, A B z, t) \diamond N(Q u, S T u$, t) $\diamond N(P z, S T u, t)$

$N(z, u, k t) \leq N(z, u, t) \diamond N(z, z, t) \diamond N(u, u, t) \diamond N(z, u, t)$

$N(z, u, k t) \leq N(z, u, t)$

By lemma, we get $z=u$. Hence, $z$ is a unique common fixed point of $A, B, S, T, P$ and $Q$. Take $B=T=I$ (Identity map), then theorem 3.1 becomes:

Corollary 3.1: Let $\left(X, M, N_{,}^{*}, \diamond\right)$ be a complete intuitionistic fuzzy metric space with $a^{*} b=\min \{a, b\}$ and $a \diamond b=\max \{a, b\}$ for all $a, b \in[0,1]$. Let $\mathrm{A}, \mathrm{S}, \mathrm{P}$ and $\mathrm{Q}$ be mappings from $\mathrm{X}$ in to itself such that the following conditions are satisfied:

(3.6) $P(X) \subseteq S(X), Q(X) \subseteq A(X)$,

(3.7) $\mathrm{P}$ or $\mathrm{A}$ is continuous,

(3.8) $(P, A)$ and $(Q, S)$ are pairs of compatible mappings of type (A), 
(3.9) there exist $k \in(0,1)$ such that for every $x, y \in X$ and $t>0$ $M(P x, Q y, k t) \geq M(A x, S y, t)$ * $M(P x, A x, t)$ * $M(Q y, S y, t)$ * $M$ $(P x, S y, t)$

$N(P x, Q y, k t) \leq N(A x, S y, t) \diamond N(P x, A x, t) \diamond N(Q y, S y, t) \diamond N(P x$, $S y, t)$

Then $A, S, P$ and $Q$ have a unique common fixed point in $X$.

\section{References}

1. Alaca C, Turkoglu D, Yildiz C. Fixed points in Intuitionistic fuzzy metric spaces. Chaos, Solitons \& Fractals. 2006; 29: 1073-1078. doi: 10.1016/j. chaos.2005.08.066

2. Atanassov K, Intuitionistic Fuzzy sets. Fuzzy sets and system. 1986; 20(1): 87-96. doi: 10.1016/\$0165-0114(86)80034-3

3. Kramosil I, Michalek J. Fuzzy metric and Statistical metric spaces. Kybernetica. 1975; 11: 326-334.

4. Menger K. Statistical metrics. Proc. Nat. Acad. Sci. 1942; 28(12): 535- 537.

5. Park JH. Intuitionistic fuzzy metric spaces. Chaos, Solitons \& Fractals. 2004; 22: 1039-1046. doi: 10.1016/j.chaos.2004.02.051

6. Schweizer B, Sklar A. Probabilistic Metric Spaces. North Holland Amsterdam. 1983. 